\title{
Análise da Performance Funcional em Indivíduos Com Instabilidade do Tornozelo: Uma Revisão Sistemática da Literatura
}

\author{
Functional Performance Assessment in Individuals with Ankle \\ Instability: A Systematic Review of the Literature
}

Eneida Yuri Suda

Rafael Novaes de Souza

Centro Universitário Capital, São

Paulo, SP.

\section{Endereço para correspondência: \\ Eneida Yuri Suda \\ Rua Masuzo Naniwa, $105-\mathrm{Bl}$. \\ Tarragona, apto 84, Bairro Mogilar \\ 08773-535 - Mogi das Cruzes, SP \\ E-mail: eyurisuda@yahoo.com.br}

Submetido em 01/07/2008

Versão final recebida em 24/10/2008

Aceito em 07/12/2008

\begin{abstract}
RESUMO
Introdução e objetivo: Os entorses do tornozelo estão entre as lesões mais frequentes nos esportes, nas atividades de vida diária, nos acidentes e nos departamentos de emergências médicas, afetando principalmente os indivíduos jovens fisicamente ativos. O objetivo deste trabalho foi realizar uma revisão sistemática da literatura a fim de levantar a existência de medidas clínicas que avaliem a performance funcional de indivíduos com instabilidade crônica do tornozelo, assim como a de estudos que verifiquem objetivamente a presença de déficits de performance funcional nesses indivíduos. Método: Foi conduzida uma busca nas bases de dados Medline, Embase e Lilacs. Essa revisão incluiu estudos que descrevem instrumentos de avaliação da performance funcional em indivíduos com instabilidade crônica do tornozelo. Os dois revisores realizaram buscas nas bases de dados computadorizadas de forma independente. Resultados: No total, seis estudos foram incluídos, apenas três considerados de alta qualidade. Os testes descritos na literatura para avaliação de performance funcional em indivíduos com instabilidade do tornozelo foram Cocontraction test, Shuttle run test, Agility hop test, Triple-crossover hop for distance, 6-m shuttle run, Figure-of-8-hop, Side hop, Up-down-hop, Single hop, Multiple hop test e Star excursion balance test. Apenas os dois últimos testes foram capazes de detectar déficits de performance funcional em indivíduos com instabilidade de tornozelo. Conclusão: Observou-se que, até o momento, apenas os testes Multiple hop test e SEBT têm sua validade e confiabilidade atestadas como ferramentas capazes de detectar déficits de performance funcional em indivíduos com instabilidade do tornozelo.
\end{abstract}

Palavras-chave: tornozelo, instabilidade articular, revisão, análise e desempenho de tarefas.

\begin{abstract}
Background and Purpose: Ankle sprains are among the commonest injuries in sports, in daily routine activities, in accidents and in medical emergency rooms, affecting mainly physically active young adults. The aim of this study was to systematically review the literature in order to identify the existence of clinical procedures to assess functional performance of individuals with chronic ankle instability, and to identify the existence of studies that objectively assess the presence of functional performance deficits in these subjects. Method: A database search was conducted in MEDLINE, EMBASE, LILACS. This review included studies that described tools that evaluated functional performance in individuals with chronic ankle instability. Two reviewers conducted the research and independently assessed the methodological quality and extracted relevant data of each included study. Results: A total of six studies were included, from which three were considered of high quality. The tests described in the literature for performance evaluation in unstable individuals were "Cocontraction test", "Shuttle run test", "Agility hop test", "Triple-hop crossover for distance," "6-m shuttle run", "Figure-of-8-hop," "Side hop,,"Up-down-hop,,"'Single hop," "Multiple hop test" and "Star excursion balance test." Only the two last tests were able to detect functional performance deficits in individuals with ankle instability. Conclusion: It was observed that until the present time, only the "Multiple Hop Test" and "SEBT" tests were valid and reliable as instruments able to detect deficits of performance in individuals with chronic ankle instability.
\end{abstract}

Keywords: ankle, joint instability, review, task performance and analysis. 


\section{INTRODUÇÃO}

Os entorses do tornozelo estão entre as lesões mais frequentes nos esportes, nas atividades de vida diária, nos acidentes e nos departamentos de emergências médicas, afetando principalmente os indivíduos jovens fisicamente ativos ${ }^{(1-4)}$. O mecanismo de lesão mais comum é uma supinação excessiva do complexo tornozelo-pé, que ocorre quando o complexo articular apresenta inversão, flexão plantar e inversão excessivas durante a descarga de peso no membro acometido (5).

Em um entorse por inversão pode ocorrer a lesão do ligamento talofibular anterior, da região ântero-lateral da cápsula articular, do ligamento calcaneofibular e do ligamento talofibular posterior, resultando na presença de dor aguda, redução da amplitude de movimento, déficit da função física(6,7).

Entre $40 \%$ e $72 \%$ das pessoas com entorse de tornozelo experimentam problemas crônicos, incluindo entorses recorrentes e limitação persistente da função ${ }^{(8,9)}$

A instabilidade do tornozelo é um fenômeno de caráter subjetivo que frequentemente se desenvolve após um entorse do tornozelo por inversão; tem sido definida como tendência do tornozelo de se deslocar durante a atividade normal; isso torna o tornozelo instável mais fraco, mais doloroso e menos funcional do que antes da lesão(10-12). As causas da instabilidade do tornozelo têm sido atribuídas a duas entidades: a instabilidade funcional e a instabilidade mecânica. A instabilidade mecânica (IM) é definida como o movimento do tornozelo além do limite fisiológico de sua amplitude de movimento, enquanto a instabilidade funcional (IF) é definida como a sensação subjetiva de instabilidade do tornozelo e/ou a presença de entorses recorrentes do tornozelo devido à presença de déficits neuromusculares e proprioceptivos ${ }^{(13)}$.

Uma vez que uma das principais características da instabilidade crônica do tornozelo é a diminuição da funcionalidade do indivíduo, levantou-se a necessidade de se avaliar o quanto essa funcionalidade pode ser afetada pela presença da instabilidade. Assim, o objetivo deste estudo é realizar uma revisão sistemática da literatura a fim de levantar a existência de medidas clínicas que avaliem a performance funcional de indivíduos com instabilidade crônica do tornozelo, assim como levantar a existência de estudos que verifiquem objetivamente a presença de déficits de performance funcional nesses indivíduos.

\section{METODOLOGIA}

\section{Busca na literatura}

Os dois revisores realizaram buscas nas bases de dados computadorizadas de forma independente. As seguintes bases de dados foram consultadas: Medline (1966 a maio de 2008), Embase (1988 a maio de 2008) e Lilacs (1966 a maio de 2008). A busca foi baseada nas combinações das palavras "tornozelo", "instabilidade", "performance funcional", "avaliação da performance", "avaliação funcional" e "teste de avaliação funcional". As referências dos artigos selecionados foram então examinadas.

\section{Tipos de estudos incluídos}

Essa revisão incluiu estudos que descrevem instrumentos de avaliação da performance funcional em indivíduos com instabilidade crônica do tornozelo. Foram considerados para essa revisão artigos completos publicados até abril de 2008 nas línguas inglesa e portuguesa. Para determinar se um estudo deveria ser incluído ou não, os resumos de todas as referências obtidas foram avaliados independentemente pelos dois revisores. Para tanto, os estudos passaram por um questionário de avaliação dos critérios de inclusão que continha duas perguntas utilizadas para delimitar a pesquisa:
1. Foram aplicados instrumentos de avaliação da performance funcional?

2. Os participantes apresentavam sinais de instabilidade no tornozelo - entorses recorrentes, falseios, dificuldades de andar e correr em superfícies irregulares, ou de realizar saltos e mudanças de direção durante a prática da atividade esportiva, ou pelo simples ato de andar?

Caso houvesse qualquer dúvida durante a avaliação dos resumos, o texto completo foi obtido e lido de forma independente por ambos os revisores. Foram incluídos apenas os estudos que responderam afirmativamente a ambas as perguntas. Caso houvesse a não concordância na avaliação dos critérios de inclusão, chegou-se a um consenso entre os dois revisores. Assim, foram incluídos nessa revisão estudos que envolveram sujeitos que apresentavam instabilidade crônica do tornozelo, seja funcional e/ou mecânica, cujos sintomas mais frequentes se apresentam como: entorses de recorrência, falseios, dificuldades de andar ou correr em superfícies irregulares, dificuldades de realizar saltos ou mudanças de direção durante a prática de atividade esportiva, ou pelo simples ato de andar ${ }^{(14)}$. Além disso, os estudos deveriam apresentavam alguma forma de avaliação da performance funcional do tornozelo.

\section{Extração dos dados}

A extração dos dados foi realizada por um dos revisores. Para cada um dos estudos selecionados identificaram-se a pergunta da pesquisa ou o objetivo do trabalho, as características da população estudada (sexo, idade, características antropométricas), a descrição dos critérios de seleção dos sujeitos com instabilidade do tornozelo e do grupo controle, quando presente, tamanho da amostra, instrumentos utilizados para a avaliação da performance funcional (definição, descrição e propriedades) e os resultados dos testes de performance.

\section{Avaliação de qualidade}

A qualidade metodológica dos estudos selecionados foi avaliada de forma independente pelos revisores utilizando um conjunto composto por cinco critérios (tabela 1). Cada critério foi classificado como positivo, negativo ou inconclusivo (informação disponível é insuficiente). Um escore total para a qualidade metodológica de cada estudo foi calculado através da soma do número de critérios que apresentaram valor positivo. Estudos com escore igual ou cima de 3 foram considerados como de "alta qualidade".

Tabela 1. Critérios utilizados para a avaliação da qualidade dos estudos

\begin{tabular}{c|l}
\hline & \multicolumn{1}{c}{ Critério } \\
\hline 1 & $\begin{array}{l}\text { Definição da amostra - critérios de inclusão para instabilidade bem } \\
\text { definidos }\end{array}$ \\
\hline 2 & $\begin{array}{l}\text { Pareamento dos grupos (no caso de comparação entre um grupo contro- } \\
\text { le e um com instabilidade) ou randomização dos grupos (no caso de com- } \\
\text { paração entre dois ou mais grupos com instabilidade de tornozelo) }\end{array}$ \\
\hline 3 & $\begin{array}{l}\text { Avaliação da validade do instrumento ou utilização de um instrumento } \\
\text { previamente validado }\end{array}$ \\
\hline 4 & $\begin{array}{l}\text { Avaliação da confiabilidade do instrumento ou utilização de um instru- } \\
\text { mento cuja confiabilidade foi previamente estabelecida }\end{array}$ \\
\hline 5 & $\begin{array}{l}\text { Avaliação da responsividade do instrumento ou utilização de um instru- } \\
\text { mento cuja responsividade foi previamente estabelecida }\end{array}$ \\
\hline
\end{tabular}




\section{RESULTADOS}

\section{Características dos estudos identificados}

A estratégia de busca da literatura forneceu 243 estudos para a seleção inicial. A partir da análise dos títulos e dos resumos dos estudos foram identificados 11 estudos relevantes, que foram selecionados para análise do manuscrito completo. Os outros estudos foram excluídos por não avaliar sujeito com instabilidade crônica do tornozelo e/ou por não apresentar em sua metodologia o uso de instrumentos que avaliassem a performance funcional desses sujeitos. Após se obter o manuscrito completo dos estudos selecionados, três estudos foram excluídos após a avaliação independente de ambos os revisores. 0 estudo realizado por Worrell et al. (1994)(15) foi excluído porque avaliou sujeitos com histórico de entorse de tornozelo, mas que não apresentavam necessariamente instabilidade deste. $\mathrm{O}$ estudo de Hals et al. (2000)(16) foi excluído porque o experimento avaliou o efeito do uso de órteses na performance funcional de indivíduos com instabilidade do tornozelo, mas não os instrumentos de avaliação da performance funcional. Da mesma forma, Hainess et al. (2004)(17) avaliou o efeito de um método de intervenção terapêutica na performance funcional, mas não os instrumentos. Assim, foram incluídos oito estudos nessa revisão (figura 1) 1 (18-23).

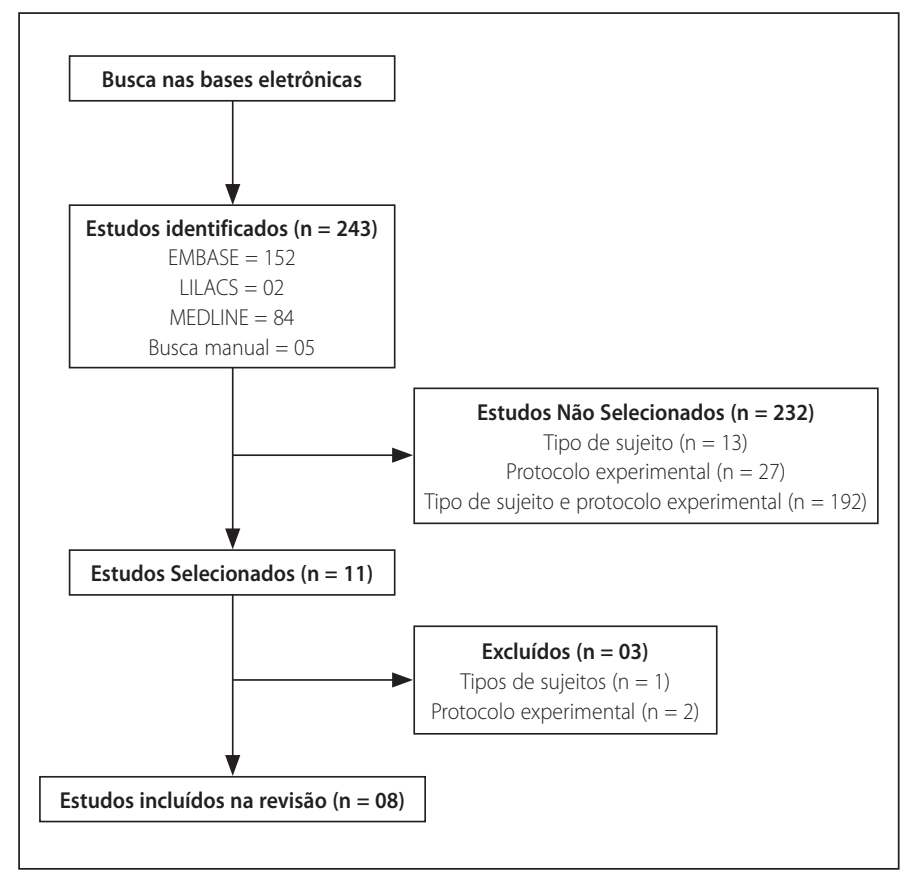

Figura 1: Seleção dos estudos pesquisados nas bases eletrônicas

As principais características dos estudos incluídos na revisão sistemática encontram-se na tabela 2.

Tabela 2. Principais características dos estudos incluídos na revisão sistemática

\begin{tabular}{|c|c|c|c|c|}
\hline Autores & Objetivo do estudo & $\begin{array}{l}\text { Tamanho da } \\
\text { amostra (n) }\end{array}$ & Características dos sujeitos & $\begin{array}{l}\text { Testes de avaliação } \\
\text { da performance } \\
\text { funcional }\end{array}$ \\
\hline Eechaute et al. & $\begin{array}{l}\text { Avaliar uma versão adaptada de um teste } \\
\text { de performance funcional (saltos múltiplos) } \\
\text { e investigar a confiabilidade e a validade dos } \\
\text { resultados em sujeitos com instabilidade crô- } \\
\text { nica do tornozelo }\end{array}$ & $\begin{array}{l}\text { Grupo Controle } \\
\text { GC) }=29 \\
\text { Grupo com } \\
\text { Instabilidade } \\
\text { Crônica } \\
(\mathrm{GIC})=29\end{array}$ & $\begin{array}{l}\text { GC - Alunos das faculdades de educação física e fisioterapia e indivíduos pro- } \\
\text { venientes de clubes locais com diferentes níveis de atividade física (recreacional } \\
\text { ou competitivo); sem história de entorse lateral de tornozelo, lesão ligamentar } \\
\text { do joelho, alterações de equilíbrio, presença de dor ou fadiga muscular nos } \\
\text { membros inferiores no momento do teste. } \\
\text { GIC - história de um entorse lateral do tornozelo traumático, queixas de entorses } \\
\text { recorrentes, presença de falseios ou diminuição do nível de performance em } \\
\text { atividades recreacionais, competitivas ou profissionais. }\end{array}$ & Multiple hop test \\
\hline Docherty et al. & $\begin{array}{l}\text { Determinar se há relação entre uma medida } \\
\text { de instabilidade funcional do tornozelo e os } \\
\text { déficits de desempenho funcional. }\end{array}$ & $\begin{array}{c}\mathrm{GC}=60 \\
\text { Grupo com } \\
\text { Instabilidade } \\
\text { Funcional } \\
(\mathrm{GIF})=42\end{array}$ & $\begin{array}{l}\text { Estudantes universitários participantes de uma associação atlética universitária } \\
\text { GC - sem história de lesões no tornozelo, fraturas ou lesões dos membros } \\
\text { inferiores } \\
\text { GIF - história de entorse de tornozelo prévio e sensação e instabilidade, avaliados } \\
\text { através de seis questões dicotômicas }\end{array}$ & $\begin{array}{l}\text { Figure-of-8-hop } \\
\text { Side hop } \\
\text { Up-down-hop } \\
\text { Single hop }\end{array}$ \\
\hline Demeritt et al. & $\begin{array}{l}\text { Determinar se o desempenho funcional está } \\
\text { prejudicado em indivíduos com instabilidade } \\
\text { crônica do tornozelo. }\end{array}$ & $\begin{array}{l}\mathrm{GC}=20 \\
\mathrm{GIC}=20\end{array}$ & $\begin{array}{l}\text { Estudantes universitários e da III Divisão e da Academia Militar } \\
\text { GC - sem história de lesões no tornozelo } \\
\text { GIC - história de pelo menos um entorse de tornozelo que o impossibilitou } \\
\text { de fazer descarga de peso no membro acometido; pelo menos um entorse de } \\
\text { repetição ou sensação de instabilidade; não estar participando de um programa } \\
\text { de reabilitação }\end{array}$ & $\begin{array}{l}\text { Cocontraction test } \\
\text { Shuttle run test } \\
\text { Agility hop test }\end{array}$ \\
\hline Munn etal. & $\begin{array}{l}\text { Verificar se os testes de performance funcional } \\
\text { são capazes de diferenciar o tornozelo com e } \\
\text { sem instabilidade em indivíduos com instabi- } \\
\text { lidade funcional unilateral de tornozelo }\end{array}$ & $\mathrm{GIF}=16$ & $\begin{array}{l}\text { Sujeitos que praticam atividades em nível de competição com história de pelo } \\
\text { menos um entorse lateral de tornozelo, sendo o ultimo episódio entre quatro se- } \\
\text { manas e um ano antes da avaliação; sintomas de fraqueza, dor ou diminuição de } \\
\text { função do membro; sem história de cirurgias ortopédicas, fraturas ou condições } \\
\text { neurológicas; sem dor ao repouso }\end{array}$ & $\begin{array}{l}\text { Triple-crossover hop } \\
\text { for distance } \\
\text { 6-m shuttle run }\end{array}$ \\
\hline Olmsted et al. & $\begin{array}{l}\text { Determinar se teste de performance funcional } \\
\text { utilizado é capaz de detectar os déficits de al- } \\
\text { cance nos sujeitos com instabilidade crônica } \\
\text { unilateral do tornozelo }\end{array}$ & $\begin{array}{l}\mathrm{GC}=20 \\
\mathrm{GIC}=20\end{array}$ & $\begin{array}{l}\text { Indivíduos da população atlética da III Divisão - nível universitário - sem história } \\
\text { de concussões cerebrais, alterações vestibulares e lesões nos membros inferiores } \\
\text { nos últimos três meses; sem infecções no ouvido, infecções respiratórias ou fria- } \\
\text { gem importante no momento do estudo; sem treinamento anterior no teste. } \\
\text { GC - sem história de lesões no tornozelo; } \\
\text { GIC - pelo menos um episódio de entorse agudo do tornozelo nas últimas seis } \\
\text { semanas; múltiplos episódios de falseio no último ano; }\end{array}$ & $\begin{array}{c}\text { Star excursion } \\
\text { balance test (SEBT) }\end{array}$ \\
\hline Hertel et al. & $\begin{array}{l}\text { (1) Verificar se é possível reduzir o número } \\
\text { de componentes do SEBT necessários para } \\
\text { detectar déficits funcionais } \\
\text { (2) Avaliar a relação entre a performance das } \\
\text { diferentes direções de alcance do teste } \\
\text { (3) Determinar quais componentes do SEBT } \\
\text { são mais afetados pela instabilidade crônica } \\
\text { do tornozelo }\end{array}$ & $\begin{array}{c}\mathrm{GC}=39 \\
\mathrm{GIC}(\text { unilateral })=48\end{array}$ & $\begin{array}{l}\text { Estudantes universitários } \\
\text { GC - sem quaisquer lesões nos membros inferiores nas últimas seis semanas } \\
\text { GIC - história de pelo menos um entorse de tornozelo que necessitou de aten- } \\
\text { dimento médico; mais de três episódios de falseio no último ano; sem história } \\
\text { de cirurgia ou fratura no membro acometido; sem história de cirurgia, fratura ou } \\
\text { lesões no tornozelo no membro contralateral }\end{array}$ & $\begin{array}{c}\text { Star excursion } \\
\text { balance test (SEBT) }\end{array}$ \\
\hline
\end{tabular}




\section{Avaliação da qualidade dos estudos incluídos}

A tabela 3 apresenta os resultados da avaliação de qualidade dos estudos incluídos. Dos seis estudos incluídos, apenas três apresentaram nível de qualidade considerado alto.

Tabela 3. Escores de qualidade dos estudos incluídos

\begin{tabular}{c|c|c|c|c|c|c|c}
\hline \multicolumn{1}{c|}{ Autores } & $\mathbf{1}$ & $\mathbf{2}$ & $\mathbf{3}$ & $\mathbf{4}$ & $\mathbf{5}$ & Escore & Qualidade \\
\hline Eechaute et al. & + & + & + & + & - & 4 & alta \\
\hline Docherty et al. & + & - & - & - & - & 1 & baixa \\
\hline Hertel et al. & + & + & + & + & + & 5 & alta \\
\hline Olmsted et al. & + & + & - & - & - & 2 & baixa \\
\hline Munn et al. & + & + & - & - & - & 2 & baixa \\
\hline Demeritt et al. & + & + & - & + & - & 3 & alta \\
\hline
\end{tabular}

Legenda: + indicativo positivo dos critérios estabelecidos; - indicativo negativo dos critérios estabelecidos.

\section{Avaliação da performance funcional}

De forma interessante, os estudos incluídos na revisão analisaram testes de performance funcional diferentes. Demeritt et al. (2002)(19) utilizaram o Cocontraction test, o Shuttle run test e o Agility hop test, não encontrando diferenças de performance entre os sujeitos com e sem instabilidade de tornozelo. Da mesma forma, Munn et al. ${ }^{(21)}$ não observaram déficits funcionais no membro com instabilidade funcional quando comparado com o contralateral saudável em indivíduos com instabilidade crônica. Os autores utilizaram os testes Triple-crossover hop for distance e 6-m shuttle run. Docherty et al. (2005) (18) observaram que indivíduos com maior grau de instabilidade apresentaram maiores déficits durante a execução dos testes Figure-of-8-hop e Side hop, não ocorrendo o mesmo com os testes Up-down-hop e Single hop.

Eechaute et al. (2008) ${ }^{(20)}$ utilizaram o Multiple hop test em seu estudo e atestaram que, além de esse teste ser capaz de detectar déficits de performance funcional em indivíduos com instabilidade de tornozelo, ele é uma ferramenta válida e confiável.

O SEBT foi avaliado em duas ocasiões ${ }^{(22,23)}$. Olmsted et al. (2002)(23) verificaram que os indivíduos com instabilidade crônica do tornozelo apresentam, de forma geral, diminuição do alcance funcional durante o teste, tanto quando se realiza a comparação entre indivíduos com instabilidade e sem, quanto quando se compara o membro acometido com o não acometido em indivíduos com instabilidade unilateral. Além disso, o alcance na direção "lateral" apresentou-se significativamente mais curto do que as outras sete direções presentes no teste (ântero-lateral, anterior, ântero-medial, medial, póstero-medial, posterior e póstero-lateral), seguido pelo ântero-lateral. Hertel et al. (2006)(22), entretanto, verificaram que a direção póstero-medial é a mais representativa para detectar déficits funcionais em sujeitos com instabilidade do tornozelo, embora as direções ântero-medial e médio-lateral também sejam capazes de detectar essas diferenças.

\section{DISCUSSÃO}

O objetivo deste trabalho foi realizar uma revisão sistemática da literatura a fim de levantar a existência de medidas clínicas que avaliem a performance funcional de indivíduos com instabilidade crônica do tornozelo, assim como levantar a existência de estudos que verifiquem objetivamente a presença de déficits de performance funcional nesses indivíduos. A instabilidade crônica do tornozelo é uma consequência frequente depois de um entorse lateral do tornozelo; estima-se que
$40 \%$ dos indivíduos que sofreram entorse inicial desenvolverão instabilidade no tornozelo ${ }^{(22)}$. Os testes de desempenho funcional devem avaliar os déficits funcionais e, consequentemente, ser capazes de discriminar o membro instável(21). Por causa da predominância da instabilidade crônica no tornozelo e as consequências causadas por ela, nosso trabalho tentou identificar estudos que apresentassem testes clínicos que viabilizam a discussão e a aplicação de testes de avaliação de performance funcional na parte clínica.

Observou-se a existência de poucos estudos sobre esse assunto. Apenas seis estudos preencheram os critérios de inclusão dessa revisão; desses, apenas três apresentaram alta qualidade metodológica ${ }^{(19,20-22)}$

O estudo de Docherty et al. (2005) $)^{(18)}$ mostrou que os testes figure-of-8-hop e side-hop foram capazes de detectar déficits funcionais em indivíduos com instabilidade. Embora esses testes tenham como componentes deslocamentos laterais, que estressam as estruturas laterais do tornozelo (side-hop), além de movimentos que levam a estresse rotacional (figure-of-8-hop) e, portanto, exigem do individuo movimentos que requerem maior estabilidade do complexo articular do tornozelo-pé, os critérios de validade, confiabilidade e responsividade não foram mensurados nesse estudo. Assim, sua aplicabilidade clínica ainda deve ser feita com ressalvas. De acordo com os próprios autores, os testes Up-down-hop e Single hop não apresentaram déficits funcionais nos indivíduos com instabilidade por englobar movimentos que são executados quase totalmente no plano sagital. Demeritt et al. (2002) ${ }^{(19)}$ não observaram a presença de déficits funcionais nos testes Cocontraction test, Shuttle run test e Agility hop test. Como todos os indivíduos avaliados eram fisicamente ativos, esses resultados sugerem que eles apresentam outras formas de compensação para o possível déficit proprioceptivo proveniente da condição de instabilidade e que os testes avaliados não foram capazes de detectá-las.

Eechaute et al. (2008)(20), entretanto, atestaram que o Multiple hop test é uma ferramenta discriminativa capaz de diferenciar pacientes com instabilidade crônica do tornozelo. Da mesma forma, Olmsted et al. (2002)(23), observaram que o SEBT é um instrumento sensível e confiável para detectar déficits de performance funcional nesses sujeitos. De forma interessante, ambos os estudos sugerem que os déficits apresentados em ambos os testes estão relacionados com uma dificuldade dos sujeitos com instabilidade de tornozelo de realizarem as correções posturais necessárias para completar as tarefas motoras requeridas, ou seja, esses sujeitos apresentariam um déficit de equilíbrio dinâmico. Recentemente, uma série de estudos detectaram alterações da atividade muscular durante a execução de atividades funcionais, como o andar(24-27) e durante a aterrissagem após a realização de saltos ${ }^{(28-30)}$. Assim, os déficits de performance funcional observados nos indivíduos que executaram os testes Multiple hop test e SEBT reforçam a ideia de que a instabilidade de tornozelo está relacionada com a presença de déficits proprioceptivos e de controle neuromuscular $(13,31,32)$.

\section{CONCLUSÃO}

Observou-se que, até o momento, apenas os testes Multiple hop test e SEBT têm sua validade e confiabilidade atestadas como ferramentas capazes de detectar déficits de performance funcional em indivíduos com instabilidade do tornozelo.

Todos os autores declararam não haver qualquer potencial conflito de interesses referente a este artigo. 


\section{REFERÊNCIAS BIBLIOGRÁFICAS}

1. Ferran NA, Maffulli N. Epidemiology of sprains of the lateral ankle ligament complex. Foot Ankle Clin 2006;11:659-62

2. Fernandez WG, Yard EE, Comstock RD. Epidemiology of lower extremity injuries among U.S. high school athletes. Acad Emerg Med 2007;14:641-5.

3. Hootman JM, Dick R, Agel J. Epidemiology of collegiate injuries for 15 sports: summary and recommendations for injury prevention initiatives. J Athl Train 2007;42:311-9.

4. Davidson PL, Chalmers DJ, Wilson BD, McBride D. Lower limb injuries in New Zealand Defence Force personnel: descriptive epidemiology. Aust N Z J Public Health 2008;32:167-73.

5. Hertel J, Denegar CR, Monroe MM, Stokes WL. Talocrural and subtalar joint instability after lateral ankle sprain. Med Sci Sports Exerc 1999;31:1501-8.

6. Safran MR, Benedetti RS, Bartolozzi AR 3rd, Mandelbaum BR. Lateral ankle sprains: a comprehensive review: part 1: etiology, pathoanatomy, histopathogenesis, and diagnosis. Med Sci Sports Exerc 1999;31:5429-37.

7. Haywood KL, Hargreaves J, Lamb SE. Multi-item outcome measures for lateral ligament injury of the ankle: a estructured review. J Eval Clin Pract 2004;10:339-52.

8. Gerber JP, Williams GN, Scoville CR, Arciero RA, Taylor DC. Persistent disability associated with ankle sprains: a prospective examination of an athletic population. Foot Ankle Int 1998;19:653-60.

9. Braun BL. Effects of ankle sprain in a general clinic population 6 to 18 months after medical evaluation. Arch Fam Med 1999;8:143-8.

10. Freeman MA. Instability of the foot after injuries to the lateral ligament of the ankle. J Bone Joint Surg Br. 1965;47:669-77.

11. Hiller CE, Refshauge KM, Bundys AC, Hebert RD, Kilbreath SL. The Cumberland ankle instability tool: a report of validity and reliability testing. Arch Phys Med Rehabil 2006;87:1235-41.

12. Monaghan $\mathrm{K}$, Delahunt $\mathrm{E}$, Caulfield $\mathrm{B}$. Ankle function during gait in patients with chronic ankle instability compared to controls. Clin Biomech 2006;21:168-74.

13. Tropp H. Commentary: Functional Ankle Instability Revisited. J Athl Train 2002;37:512-5.

14. Suda EY. Análise eletromiográfica comparativa de tornozelo durante a aterrissagem em jogadores de vôlei com instabilidade crônica [dissertação]. São Paulo: Faculdade de Medicina, Universidade de São Paulo; 2006. 100p.

15. Worrell TW, Booher LD, Hench KM. Closed kinetic chain assessment following inversion ankle sprain. J Sport Rehabil 1994;3:197-203

16. Hals TMV, Sitler MR, Mattacola CG. Effect of a semi-rigid ankle stabilizer on performance in persons with functional ankle instability. J Orthop Sports Phys Ther 2000;30.
17. Hainess KL, Hargreaves J, Lamb SE. Multi-item outcome measures for lateral ligament injury of the ankle: a structured review. J Eval Clin Prac 2004;10:339-52.

18. Docherty CL, Arnold BL, Gansneder BM, Hurwitz S, Gieck J. Functional-performance deficits in volunteers with functional ankle instability. J Athl Train 2005;40:30-4.

19. Demeritt KM, Shultz SJ, Docherty CL, Gansneder BM, Perrin DH. Chronic ankle instability does not affect lower extremity functional performance. J Athl Train 2002;37:507-11.

20. Eechaute $C$, Vaes $P$, Duquet $V$. Functional performance deficits in patients with cai: validity of the multiple hop test. Clin J Sport Med 2008;18:124-9.

21. Munn J, Beard DJ, Refshauge KM, Lee RWY. Do functional-performance tests detect impairment in subjects with ankle instability? J Sport Rehabil 2002;11:40-50.

22. Hertel J, Braham RA, Hale SA, Olmsted-kramer LC. Simplifying the star excursion balance test: analyses of subjects with and without chronic ankle instability. J Orthop Sports Phys Ther 2006;36:131-7.

23. Olmsted LC, Carciat CR, Hertel J, Shultz SJ. Efficacy of the star excursion balance tests in detecting reach deficits in subjects with chronic ankle instability. J Athl Train 2002;37:501-6.

24. Louwerens JW, van Linge B, de Klerk LW, Mulder PG, Snijders CI. Peroneus longus and tibialis anterior muscle activity in the stance phase. A quantified electromyographic study of 10 controls and 25 patients with chronic ankle instability. Acta Orthop Scand 1995;66:517-23.

25. Santilli V, Frascarelli MA, Paoloni M, Frascarelli F, Camerota F, De Natale L, et al. Peroneus longus muscle activation pattern during gait cycle in athletes affected by functional ankle instability: a surface electromyographic study. Am J Sports Med 2005;33:1183-7.

26. Delahunt E, Monaghan K, Caulfield B. Altered neuromuscular control and ankle joint kinematics during walking in subjects with functional instability of the ankle joint. Am J Sports Med 2006;34:1970-6.

27. Monaghan K, Delahunt E, Caulfield B. Ankle function during gait in patients with chronic ankle instability compared to controls. Clin Biomech (Bristol, Avon) 2006;21:168-74.

28. Delahunt E, Monaghan K, Caulfield B. Changes in lower limb kinematics, kinetics, and muscle activity in subjects with functional instability of the ankle joint during a single leg drop jump. J Orthop Res 2006;24:1991-2000.

29. Santos MJ, Liu H, Liu W. Unloading reactions in functional ankle instability. Gait Posture 2008;27:589-94.

30. Suda EY, Amorim CF, de Camargo Neves Sacco I. Influence of ankle functional instability on the ankle electromyography during landing after volleyball blocking. J Electromyogr Kinesiol 2007;4. [Epub ahead of print]

31. (b)Freeman MA, Dean MR, Hanham IW. The etiology and prevention of functional instability of the foot. J Bone Joint Surg Br 1965:47:678-85.

32. Hertel J. Functional instability following lateral ankle sprain. Sports Med 2000;29:361-71. 